\title{
Fulminant Community-Acquired Hemorrhagic Pneumonia due to Pseudomonas aeruginosa in a Patient With Anorexia Nervosa
}

\author{
Yuki Kotani ${ }^{\mathrm{a}, \mathrm{c}}$, Kenji Kubo ${ }^{\mathrm{b}}$, Toshihide Tsujimoto ${ }^{\mathrm{a}}$
}

\begin{abstract}
Community-acquired pneumonia (CAP) due to Pseudomonas aeruginosa is rare. We report a case of a 29 -year-old female with anorexia nervosa admitted to our intensive care unit for CAP due to $P$. aeruginosa. Although she had no previously known pseudomonal risk factors, gram-negative rods found in the sputum Gram stain led to immediate administration of an antipseudomonal drug. Despite intensive care, she died on day 4 of hospitalization. Anorexia nervosa can be a risk factor for CAP due to P. aeruginosa. Gram-negative rods in the sputum of patients with severe CAP can be a key to choose antipseudomonal drugs.
\end{abstract}

Keywords: Community-acquired pneumonia; Pseudomonas aeruginosa; Gram stain; Intensive care

\section{Introduction}

Pseudomonas aeruginosa is a rare pathogen of communityacquired pneumonia (CAP) [1-4]. CAP due to this pathogen is associated with higher cases of inappropriate initial therapy and mortality [2]. Most patients suffering from CAP due to $P$. aeruginosa were reported to have baseline risk factors, such as previous hospital admission and pulmonary comorbidity [5]. While there are several case reports on CAP due to $P$. aeruginosa in previously healthy hosts [6-8], only one report described a patient with anorexia nervosa [9]. Here, we report a case of fulminant CAP due to $P$. aeruginosa in young woman with anorexia nervosa who died within several days.

Manuscript accepted for publication May 12, 2017

aDepartment of Critical Care Medicine, Japanese Red Cross Society Wakayama Medical Center, Wakayama City, Wakayama Prefecture, Japan

bepartment of Infectious Diseases, Japanese Red Cross Society Wakayama Medical Center, Wakayama City, Wakayama Prefecture, Japan

${ }^{\mathrm{c} C}$ Corresponding Author: Yuki Kotani, Japanese Red Cross Society Wakayama Medical Center, 4-20, Komatsubara-dori, Wakayama City, Wakayama Prefecture,640-8558, Japan. Email: dkivoar287@gmail.com

doi: https://doi.org/10.14740/jmc2833w

\section{Case Report}

A 29-year-old woman presented to our emergency department with general malaise. She was in good health until the previous day. Her past medical history included anorexia nervosa for 11 years, and her body mass index was $11 \mathrm{~kg} / \mathrm{m}^{2}$. She had never been a smoker and had no family history of tuberculosis. She denied using any hot tubs, whirlpools or home humidifiers. Her vital signs were as follows: heart rate, 65 beats per minute; blood pressure, $80 / 55 \mathrm{~mm} \mathrm{Hg}$; respiratory rate, 24 breaths/ min; oxygen saturation, 98\% (room air) and temperature, 35.9 ${ }^{\circ} \mathrm{C}$. On physical examination, no rale was heard but her sputum was bloody.

Laboratory tests showed remarkable leukocytopenia $(1,300$ cells $/ \mu \mathrm{L})$ and thrombocytopenia $(60,000$ cells $/ \mu \mathrm{L})$. The result of the human immunodeficiency virus serologic test was negative. Beta-D glucan measurement was negative. The chest X-ray and computed tomography (CT) showed consolidation in the right upper lobe with a cavity (Fig. 1a, b). In the $6 \mathrm{~h}$ since her arrival in the emergency department, her respiration deteriorated rapidly. She was admitted to the intensive care unit (ICU) and intubated.

She was diagnosed to have septic shock due to CAP. The sputum Gram stain showed numerous gram-negative rods, few gram-positive cocci, few gram-positive rods and rare leukocytes (Fig. 2). The urinary antigens for Streptococcus pneumoniae and Legionella pneumophila were negative.

She received meropenem and pazufloxacin, considering the possibility of CAP due to $P$. aeruginosa. High doses of norepinephrine and vasopressin were necessary to treat severe hypotension. Continuous renal replacement therapy was initiated for hyperkalemia and progressive metabolic acidosis. During the first $8 \mathrm{~h}$ after admission, airway hemorrhage as much as 1,400 mL occurred. Bronchoscopy showed massive hemorrhage coming up to the tracheal tube, which required massive transfusion and high positive end expiratory pressure $(18 \mathrm{~cm}$ $\mathrm{H}_{2} \mathrm{O}$ ). As the dynamic CT scan revealed no extravasation and the hemorrhage ceased until day 2 of hospitalization, bronchial artery embolization was not performed.

Despite the intensive care mentioned above, the patient's condition worsened progressively. The consolidation on the chest radiograph was localized to the right upper field on admission but extended to almost all lung fields (Fig. 3). She died on day 4 of hospitalization. The blood and sputum cultures showed 

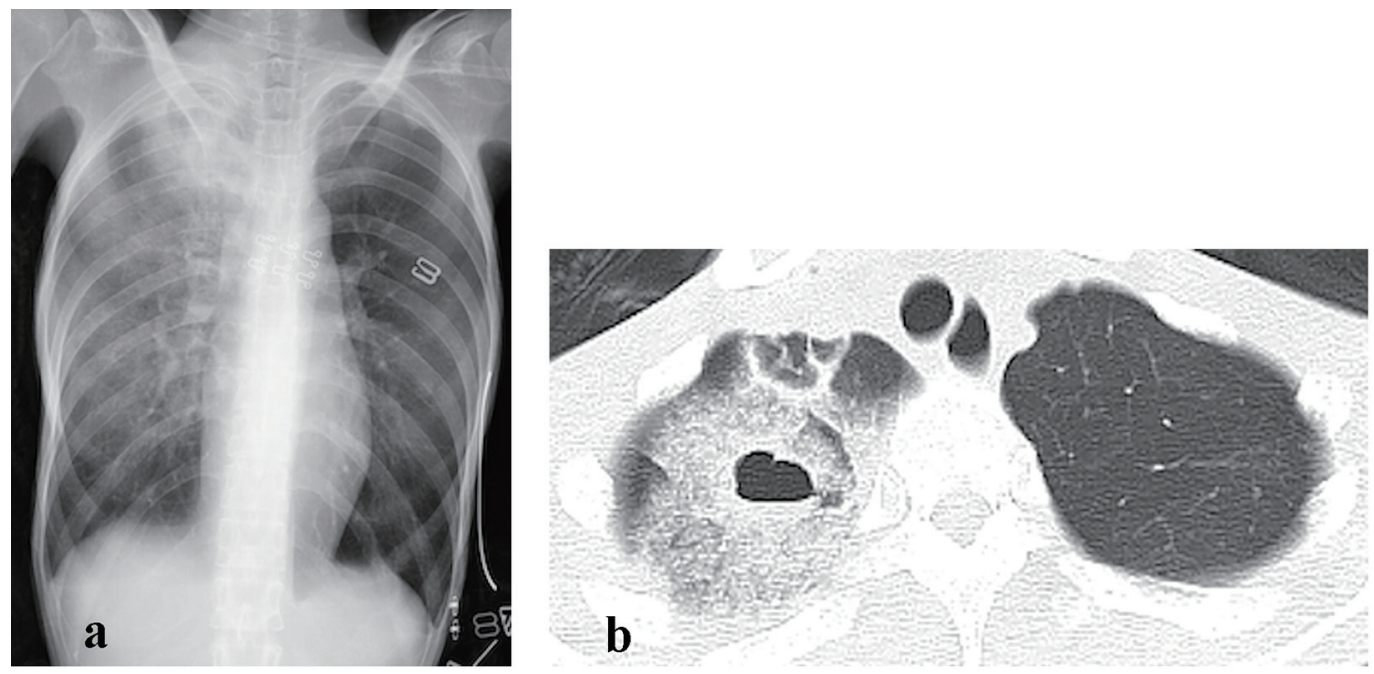

Figure 1. The chest X-ray (a) and computed tomography $(\mathrm{CT})(\mathrm{b})$ on admission showed consolidation in the right upper lobe with a cavity.

P. aeruginosa susceptible to meropenem and pazufloxacin.

\section{Discussion}

This case suggested two clinically important issues. Anorexia nervosa can be a risk factor for CAP due to P. aeruginosa. Gram-negative rods found in the sputum Gram stain can lead to immediate administration of antipseudomonal drugs in CAP patients requiring admission to the ICU.

Firstly, anorexia nervosa can be a risk factor for CAP due to $P$. aeruginosa. P. aeruginosa is a rare pathogen that causes CAP [1-4]. Several risk factors have been reported for CAP due to $P$. aeruginosa such as probable aspiration, previous hospital admission, previous use of antibiotics, pulmonary comorbidity, male sex and pneumonia severity index risk class IV to $\mathrm{V}[2,5]$. Although the present case had no known risk factors listed above, she had suffered from anorexia nervosa

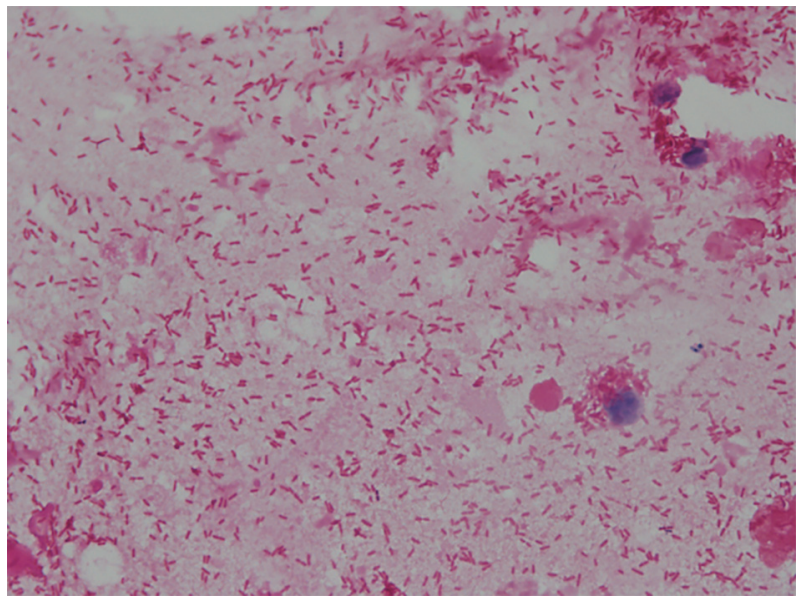

Figure 2. The sputum Gram stain on admission showed numerous gram-negative rods and rare leukocytes. for 11 years. There are several reports of patients with anorexia nervosa who developed pneumonia due to P. aeruginosa [9], pulmonary aspergillosis $[10,11]$ or mycobacterial infections [12]. Because malnutrition from anorexia nervosa has negative effects on immune system [13], anorexia nervosa may be a risk factor of CAP due to P. aeruginosa.

Several cases of CAP due to P. aeruginosa in patients without these risk factors have also been reported [6-8]. A study using the administrative database of the Department of Veterans Affairs showed that only one-third of patients with $\mathrm{CAP}$ due to $P$. aeruginosa had at least one of the following risk factors: prior oral corticosteroid use in COPD, prior antibiotic use and bronchiectasis [14].

In terms of severity, the condition of the presented patient

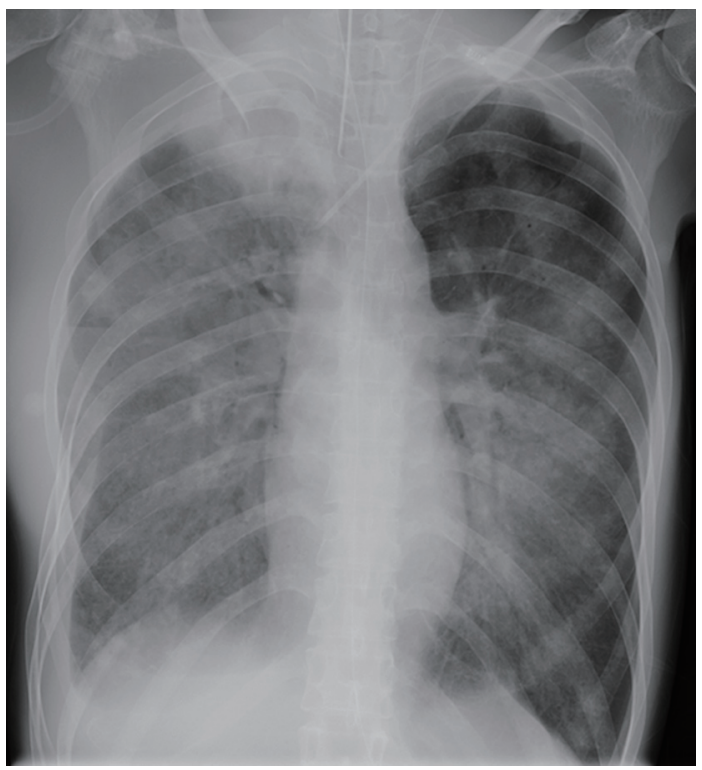

Figure 3. The consolidation extended to almost all lung fields on the day 4 of hospitalization. 
was fulminant. Mortality was higher in patients with CAP due to $P$. aeruginosa than that in patients with CAP due to other pathogens $[2,5]$. Among patients with CAP due to $P$. aeruginosa, a fulminant variant in previously healthy patients was reported [8]. This review of the literature on P. aeruginosa CAP in 12 previously healthy adults showed that the mortality rate was $33 \%$, and the median time between hospital admission and death was only $11 \mathrm{~h}$ among the non-survivors. Although the mechanism causing a fatal variant of CAP due to $P$. aeruginosa is uncertain, necrotizing pneumonia with gram-negative rods invading the walls of alveolar blood vessels may contribute to its rapid progression $[6,8]$. The present case had massive hemoptysis, suggesting necrotizing pneumonia.

Secondly, gram-negative rods on the sputum Gram stain can lead to immediate administration of antipseudomonal drugs in CAP patients requiring admission to the ICU. The current guidelines for CAP stratify initial antimicrobial therapies according to $P$. aeruginosa risk factors [15]. As mentioned above, risk factors for CAP due to this pathogen, however, have not been completely understood. As a result, many patients with CAP due to $P$. aeruginosa receive inappropriate empiric antimicrobial treatment, resulting in high mortality rates [2].

Although a sputum Gram stain may be a useful tool to decide the initial antimicrobial treatment in CAP, the Infectious Diseases Society of America/American Thoracic Society guidelines on CAP recommend a pre-treatment Gram staining of sputum only if a good quality sputum specimen can be obtained and the quality performance measures for collection, transport and processing of samples can be met. In the present case of severe CAP without known risk factors for $P$. aeruginosa, the gram-negative rods in sputum were a key to choose an antipseudomonal drug. A case report on two cases of fulminant CAP due to $P$. aeruginosa suggested that gramnegative rods in sputum microscopy should be considered as a clue for an early diagnosis [8]. A recent study [16] assessing the usefulness of sputum Gram staining in the etiologic diagnosis of CAP showed that sputum Gram staining was highly specific for CAP due to $P$. aeruginosa $(99.8 \%)$ in spite of the low sensitivity $(22.2 \%)$. The result indicated that physicians should consider using antipseudomonal drugs when gramnegative rods are observed in the sputum of a patient with CAP regardless of the presence or absence of risk factors for $P$. aeruginosa.

The patient in the present case died within 4 days despite administration of antipseudomonal drugs. Although antimicrobial treatments have a limited capacity to alter the clinical course of severe infections, early and adequate antibiotics are recommended [17].

\section{Conflicts of Interest}

No potential conflicts of interest relevant to this article were reported.

\section{References}

1. Gadsby NJ, Russell CD, McHugh MP, Mark H, Con- way Morris A, Laurenson IF, Hill AT, et al. Comprehensive Molecular Testing for Respiratory Pathogens in Community-Acquired Pneumonia. Clin Infect Dis. 2016;62(7):817-823.

2. Cilloniz C, Gabarrus A, Ferrer M, Puig de la Bellacasa J, Rinaudo M, Mensa J, Niederman MS, et al. Community-Acquired Pneumonia Due to Multidrug- and NonMultidrug-Resistant Pseudomonas aeruginosa. Chest. 2016;150(2):415-425.

3. Cilloniz C, Ewig S, Polverino E, Marcos MA, Esquinas C, Gabarrus A, Mensa J, et al. Microbial aetiology of community-acquired pneumonia and its relation to severity. Thorax. 2011;66(4):340-346.

4. Song JH, Oh WS, Kang CI, Chung DR, Peck KR, Ko $\mathrm{KS}$, Yeom JS, et al. Epidemiology and clinical outcomes of community-acquired pneumonia in adult patients in Asian countries: a prospective study by the Asian network for surveillance of resistant pathogens. Int J Antimicrob Agents. 2008;31(2):107-114.

5. Arancibia F, Bauer TT, Ewig S, Mensa J, Gonzalez J, Niederman MS, et al. Community-acquired pneumonia due to gram-negative bacteria and pseudomonas aeruginosa: incidence, risk, and prognosis. Archives of Internal Medicine. 2002;162(16):1849-1858.

6. Hatchette TF, Gupta R, Marrie TJ. Pseudomonas aeruginosa community-acquired pneumonia in previously healthy adults: case report and review of the literature. Clin Infect Dis. 2000;31(6):1349-1356.

7. Ishihara S, Takino M, Okada Y, Mimura K. Septic shock due to Pseudomonas aeruginosa in a previously healthy woman. Intensive Care Med. 1995;21(3):226-228.

8. Henderson A, Kelly W, Wright M. Fulminant primary Pseudomonas aeruginosa pneumonia and septicaemia in previously well adults. Intensive Care Med. 1992;18(7):430-432.

9. Ugajin M, Miwa S, Suda T, Shirai M, Hayakawa H, Chida K. [A case of pulmonary infection caused by Pseudomonas aeruginosa with anorexia nervosa]. Nihon Kokyuki Gakkai Zasshi. 2009;47(12):1126-1130.

10. Mogi A, Kosaka T, Yamaki E, Kuwano H. Pulmonary aspergilloma in patient with anorexia nervosa: case report. Ann Thorac Cardiovasc Surg. 2012;18(5):465-467.

11. Shimoni Z, Goldenberg A, Niven M. Fatal invasive pulmonary aspergillosis presenting as profound hypoglycemia in a patient with anorexia nervosa. Eur J Intern Med. 2006;17(4):295-297.

12. Hotta M, Nagashima E, Takagi S, Itoda I, Numata T, Kobayashi N, Takano K. Two young female patients with anorexia nervosa complicated by Mycobacterium tuberculosis infection. Intern Med. 2004;43(5):440-444.

13. Marcos A. Eating disorders: a situation of malnutrition with peculiar changes in the immune system. Eur J Clin Nutr. 2000;54(Suppl 1):S61-64.

14. Sibila O, Laserna E, Maselli DJ, Fernandez JF, Mortensen EM, Anzueto A, Waterer G, et al. Risk factors and antibiotic therapy in P. aeruginosa community-acquired pneumonia. Respirology. 2015;20(4):660-666.

15. Mandell LA, Wunderink RG, Anzueto A, Bartlett JG, Campbell GD, Dean NC, Dowell SF, et al. Infectious 
Diseases Society of America/American Thoracic Society consensus guidelines on the management of community-acquired pneumonia in adults. Clin Infect Dis. 2007;44(Suppl 2):S27-72.

16. Fukuyama H, Yamashiro S, Kinjo K, Tamaki H, Kishaba T. Validation of sputum Gram stain for treatment of community-acquired pneumonia and healthcare-associated pneumonia: a prospective observational study. BMC Infect Dis. 2014;14:534.

17. Rhodes A, Evans LE, Alhazzani W, Levy MM, Antonelli M, Ferrer R, Kumar A, et al. Surviving Sepsis Campaign: International Guidelines for Management of Sepsis and Septic Shock: 2016. Intensive Care Med. 2017;43(3):304377. 